\title{
Unitarized pion-nucleon scattering within heavy baryon chiral perturbation theory
}

\author{
A. Gómez Nicola and J. R. Peláez \\ Departamento de Física Teórica, Universidad Complutense, 28040 Madrid, Spain
}

(Received 27 December 1999; published 6 June 2000)

\begin{abstract}
By means of the inverse amplitude method we unitarize the elastic pion-nucleon scattering amplitudes obtained from heavy baryon chiral perturbation theory to $\mathcal{O}\left(q^{3}\right)$. Within this approach we can enlarge their applicability range and generate the $\Delta(1232)$ resonance. We can find a reasonable description of the pion nucleon phase shifts with $\mathcal{O}\left(q^{2}\right)$ parameters in agreement with the resonance saturation hypothesis. However, the uncertainties in the analysis of the low energy data as well as the large number of chiral parameters, which can have strong correlations, allow us to obtain very good fits with rather different sets of chiral constants.
\end{abstract}

PACS number(s): 12.39.Fe, 12.38.Cy, 13.75.Gx, 13.85.Dz

Chiral symmetry plays a fundamental role in the interactions between pions and nucleons. However, in order to go beyond current algebra or tree level calculations from simple models, one needs an effective low-energy field theory with all the QCD symmetries and a systematic power counting. Heavy baryon chiral perturbation theory (HBChPT) [1] is the best candidate to date for such a theory. For $S U(2)$ chiral symmetry, the HBChPT degrees of freedom are the nucleons and pions. The pions are the Nambu-Goldstone bosons of the spontaneous chiral symmetry breaking, whereas the nucleons are included as an isospin doublet. In the case of $S U(3)$ symmetry, the pseudoscalar meson and baryon octets are required to describe the meson-baryon sector. There are also non-minimal formulations considering the baryon decuplet as a fundamental field [2].

HBChPT is built as an expansion in derivatives and meson masses, including all terms compatible with chiral symmetry. It follows the same philosophy as chiral perturbation theory (ChPT) in the purely mesonic sector [3]. However, there are two important differences between them. First, the scale in the HBChPT expansion is not just the chiral symmetry breaking scale of pion loops $\Lambda_{\chi}=4 \pi f_{\pi} \simeq 1.2 \mathrm{GeV}$, but also the mass of the nucleons $m_{B} \simeq 1 \mathrm{GeV}$. Second, the nucleon four-momentum is of the same order as the expansion scale, no matter how small is the momentum transfer and even in the chiral limit [4]. HBChPT circumvents this problem by treating $m_{B}$ as large compared to the external momenta and redefining the nucleon fields in terms of velocity-dependent eigenstates satisfying a massless Dirac equation. The antibaryon components can be integrated out [5]. Once this is done, it is possible to find a systematic power counting in $k / \Lambda_{\chi}, k / m_{B}, q / \Lambda_{\chi}$ and $q / m_{B}$, where $q$ stands for any meson mass or external momentum, generically denoted by $\mathcal{O}(q)$. With the effective vertices up to a given order, one can calculate loop diagrams. Each loop increases the order of the diagram so that any divergence can be absorbed by renormalizing the coefficients of higher order operators. It is thus possible to obtain finite results order by order for any observable, but paying the price of introducing more chiral parameters. In particular, the full HBChPT Lagrangian up to $\mathcal{O}\left(q^{3}\right)$ has been given in [6,7]. It involves 5 unknown coefficients to $\mathcal{O}\left(q^{2}\right)$ and 23 more to $\mathcal{O}\left(q^{3}\right)$, which have to be fitted to experiment. In this paper, we will concentrate on the pion-nucleon scattering amplitude derived from $S U(2)$ HBChPT to $\mathcal{O}\left(q^{3}\right)$, which we will unitarize using the inverse amplitude method (IAM).

Despite its difficulty, several works related to $\pi N$ scattering within HBChPT have appeared in the literature [7-9]. Here we will follow the notation of the first $\mathcal{O}\left(q^{3}\right)$ complete result [9]. Thus, we denote by $a_{i}$ and $b_{i}$ the $\mathcal{O}\left(q^{2}\right)$ and $\mathcal{O}\left(q^{3}\right)$ parameters, respectively. The translation to the notation of [7,8] can be found in [7]. Only four $\mathcal{O}\left(q^{2}\right)$ and five $\mathcal{O}\left(q^{3}\right)$ combinations of chiral parameters are relevant for $\pi-N$ scattering. There are two sets of fitted parameters in the literature: in [9], the extrapolated threshold values of [10], together with the nuclear $\sigma$-term and the GoldbergerTreiman discrepancy were taken as the experimental input, whereas in [7], the $S$ and $P$-wave phase shifts, somewhat away from threshold, were used as data for the fits. These phase shifts are given in [11] and are obtained as extrapolations from experimental data. Note that in [12] it is suggested that the data in [11] yield a too large $\sigma$ term when analyzed with HBChPT. As their authors pointed out, both procedures are subject to some caveats, either because of the many uncertainties of the data near threshold (see [7]), or because the errors for the extrapolated phase shifts are clearly underestimated (see [9]). Concerning theoretical estimates, in [8] it was suggested that the $a_{i}$ could be understood from resonance exchange saturation. Further constraints from dispersive techniques can be found in [12]. In general, there is a fairly good agreement between the $a_{i}$ values, but that is not the case for $b_{i}$ (see [12]).

We should stress that the predictions obtained within this framework are promising, although not as impressive [9] as those of ChPT for mesons. HBChPT is, of course, limited to low pion momentum and, with the presently available calculations, certainly below $q_{\pi} \lesssim 200 \mathrm{MeV}$ [7]. The reason is, basically, that the convergence of HBChPT is rather slow. As a matter of fact, the contributions of the first three orders are frequently comparable.

In order to improve this situation, one could go to the next order, thus dealing with many more parameters. We could also introduce more degrees of freedom, like the lightest resonances [13], but that would also increase the number of parameters. In addition, some kind of unitarization should also be carried out in that case, to impose strict unitarity. Recently [14], remarkable results have been obtained by uni- 
tarizing with the N/D method the lowest order HBChPT at tree level, including explicitly the $\Delta(1332)$ and $N^{*}(1440)$ resonances.

We propose an alternative approach. Encouraged by the HBChPT results and the success of unitarization in mesonmeson scattering $[15,16]$, we will unitarize the amplitude without introducing additional fields.

\section{A. The IAM applied to $\pi-N$ scattering}

Unitarization is not foreign to effective theories. In fact, Padé approximants with very simple models are enough to describe the main features of $\pi-N$ scattering [18]. Although a systematic application within an effective Lagrangian approach was called for, it was never carried out.

Customarily [17], the data are presented in terms of partial waves of definite isospin $I$, orbital angular momentum $L$ and total angular momentum $J$, using the spectroscopic notation $L_{2 I+1,2 J+1}$ (with $L=S, P, \ldots$ waves). Generically, the HBChPT $\pi-N$ partial waves, $t$, are obtained as a series in the momentum transfer and meson masses. Thus, basically, they are polynomials in the energy and mass variables (as well as logarithms from the loops, which provide the cuts and imaginary parts required by unitarity). Such an expansion will never satisfy the $\pi$-N elastic unitarity condition

$$
\operatorname{Im} t=q_{c m}|t|^{2} \Rightarrow \operatorname{Im} \frac{1}{t}=-q_{c m} \Rightarrow \frac{1}{t}=\operatorname{Re} \frac{1}{t}-i q_{c m},
$$

with $q_{c m}$ the center of mass momentum of the incoming pion. But HBChPT satisfies unitarity perturbatively; i.e.,

$$
\operatorname{Im} t_{1}=\operatorname{Im} t_{2}=0 ; \quad \operatorname{Im} t_{3}=q_{c m}\left|t_{1}\right|^{2}, \ldots,
$$

where $t_{k}$ stands for the $\mathcal{O}\left(q^{k}\right)$ contribution to the amplitude. This is indeed the case in [7], but not in [9], where an additional redefinition of the nucleon field allows to eliminate the $(v \cdot \nabla)^{2} / 2 m$ terms in the Lagrangian [6]. We have performed an additional $1 / m$ expansion of the results in [9] in order to recover a pure expansion satisfying Eq. (2). Thus, we count $q_{c m}$ and $M$ as $\mathcal{O}(\epsilon)$, so that each partial wave reads $t \simeq t_{1}$ $+t_{2}+t_{3}+\mathcal{O}\left(\epsilon^{4}\right)$, where the subscript stands for the order $\epsilon$ of each contribution. We have then checked that Eq. (2) is verified.

However, from Eq. (1) any unitarity elastic amplitude has exactly the following form:

$$
t=\frac{1}{\operatorname{Re}(1 / t)-i q_{c m}},
$$

for physical values of the energy, and below any inelastic threshold. The problem, of course, is how to obtain $\operatorname{Re}(1 / \mathrm{t})$. For instance, setting $\operatorname{Re}(1 / t)=\left|q_{c m}\right| \cot \delta=-1 / a+\left(r_{0} / 2\right) q_{c m}^{2}$ we reobtain the familiar effective range approximation, whereas by taking $\operatorname{Re} t \simeq t_{1}$ we arrive at a LippmannSchwinger like equation [16]. A frequent criticism to unitarization is its apparent arbitrariness, although from Eq. (3) we see that the difference between two unitarization methods is the way of approximating $\operatorname{Re}(1 / t)$. Since we want to restrict our Lagrangian to include just pions and nucleons pre- serving the $S U(2)$ chiral symmetry, the most general approximation to $\operatorname{Re}(1 / \mathrm{t})$ is HBChPT. In particular, we will take the $\mathcal{O}\left(q^{3}\right)$ calculations, but the method can be easily generalized to higher orders. Thus, we arrive at

$$
t \simeq \frac{t_{1}^{2}}{t_{1}-t_{2}+t_{2}^{2} / t_{1}-\operatorname{Re} t_{3}-i q_{c m} t_{1}^{2}},
$$

where we have only kept the relevant order in $\operatorname{Re}\left[\left(t_{1}+t_{2}\right.\right.$ $\left.\left.+t_{3}\right)^{-1}\right]$. This is the $\mathcal{O}\left(q^{3}\right)$ form of the IAM. Note that if we reexpand in powers of $q$, we recover at low energies the HBChPT result. However, as it is written, the amplitude explicitly satisfies elastic unitarity. Furthermore, using Eq. (2), we can rewrite $\operatorname{Re} t_{3}+i q_{c m} t_{1}^{2}=t_{3}$, which can be analytically continued to the complex plane [where, for instance, we will look for the pole associated to the $\Delta(1232)]$. Incidentally, Eq. (4) thus rewritten is a Padé approximant of the $\mathcal{O}\left(q^{3}\right)$ series. From the $\mathrm{K}$ matrix point of view, we have identified $K^{-1}=\operatorname{Re} t^{-1}$.

Even though the elastic unitarity condition is only satisfied for real values of $s$ above threshold, the use of the IAM in the complex plane can be justified using dispersion relations [15], provided one is not very far from the physical cut. In other regions (around the left cut for instance) the IAM would be inappropriate. As a consequence, it is also possible to reproduce the poles in the second Riemann sheet, which are close to the physical cut and are associated to resonances. As a matter of fact the IAM has been successfully applied to meson-meson scattering [15]. In particular, using the $\mathcal{O}\left(p^{4}\right)$ ChPT Lagrangian, the IAM generalized to coupled channels yields a remarkable description of all channels up to 1.2 $\mathrm{GeV}$, including seven resonances [16]. Using the LippmannSchwinger like equation mentioned above it is also possible to describe the S-wave kaon-nucleon scattering, using the lowest order Lagrangian [19], including the $\Lambda$ (1405).

Let us then use Eq.(4) when $t$ are the $L_{2 I+1,2 J+1} \pi-N$ partial waves. The resulting amplitudes will be fitted to the [11] phase shifts, which are actually an extrapolation, not including the experimental errors. For the fit we have used the MINUIT function minimization and error analysis routine from the CERN program Library. As it is customarily done in the literature, we will assign an error to the data in [11]. For instance, in Ref. [7] the central points have been given a $3 \%$ uncertainty. However, since our fits will cover wide energy ranges, the use of a constant relative error will give more weight to the low energy data. Thus we have also added an additional systematic error of 1 degree. (A 5\% error plus a $\sqrt{2}$ systematic error was used in [14].) This error is needed to use the minimization routine, and, although the order of magnitude may seem appropriate, the values are rather arbitrary, so that the meaning of the $\chi^{2} / \mathrm{DOF}$ obtained from MINUIT has to be interpreted cautiously.

Furthermore, the data near threshold are subject to many uncertainties, so that, also following [7], we will start our fits at $\sqrt{s}=1130 \mathrm{MeV}$. Hence the threshold parameters are real predictions in our approach. In addition, we should limit the approach to energies where inelasticities can be neglected. In particular, we will not use our $P_{11}, P_{13}$ and $P_{31}$ phase shifts 
TABLE I. IAM results. In fit 1 , we keep the $\mathcal{O}\left(q^{2}\right)$ values of [9]. In fit 2 , the $a_{i}$ are constrained to the ranges predicted by resonance saturation [8]. Due to the strong correlations (see text) only certain $\hat{b}_{i}$ combinations could be meaningful.

\begin{tabular}{lccccccccc}
\hline \hline & $a_{1}$ & $a_{2}$ & $a_{3}$ & $a_{5}$ & $\hat{b}_{1}+\hat{b}_{2}$ & $\hat{b}_{3}$ & $\hat{b}_{6}$ & $\hat{b}_{16}-\hat{b}_{15}$ & $\hat{b}_{19}$ \\
\hline Fit 1 & -2.6 & 1.4 & -1.0 & 3.3 & 28.1 & -29.8 & 2.1 & 33.3 & 12.9 \\
Fit 2 & -2.1 & 1.3 & -0.8 & 3.6 & 22.3 & -26.1 & 2.5 & 26.7 & 10.1 \\
\hline \hline
\end{tabular}

above the $\pi \pi N$ threshold $(\sqrt{s} \simeq 1220 \mathrm{MeV}$ ), since they are very small and inelasticities could be significant. The $S_{31}$ and $S_{11}$ phase shifts are larger and we fitted them up to $\simeq 1360 \mathrm{MeV}$. The inelasticities in $P_{33}$ are negligible up to $1400 \mathrm{MeV}$ since this channel is dominated by the $\Delta(1232)$ which is strongly coupled to $\pi-N$.

(a) The IAM and resonance saturation. Following the suggestion that the $O\left(p^{2}\right)$ parameters can be understood from resonance saturation [8], it is natural to try to make an IAM fit constrained with this hypothesis. Thus, we first fix the $a_{i}$ to the values of [9], which are compatible with the saturation hypothesis. The resulting $O\left(p^{3}\right)$ parameters are given in Table I. In addition, we give in Table I the values for a second fit where we have allowed the $a_{i}$ parameters to vary within the ranges expected from resonance saturation. The results of fit 2 are plotted, as a solid line, in Fig. 1. We used "hatted"' quantities, $\hat{b}_{i}$, because their values do not necessarily correspond to those of HBChPT since now they are also absorbing the IAM resummation effects and some high energy information. Only if there was a very good convergence of the theory at low energies the values of $b_{i}$ should be similar to the $\hat{b}_{i}$ (as it happens in ChPT). Therefore, at present, our $\hat{b}_{i}$ should not be used to calculate any other process at low energies.

Not surprisingly, there are strong correlations between parameters. Unfortunately, from MINUIT we cannot get the actual form of the correlation. However, looking only at linear combinations with integer coefficients, some of them, like $\hat{b}_{1}+\hat{b}_{2}+\hat{b}_{3}$ or $\hat{b}_{1}+\hat{b}_{2}+2 \hat{b}_{3}+\hat{b}_{16}-\hat{b}_{15}$, remain within natural sizes for these fits. Nevertheless, as we have commented, there is a considerable uncertainty in the precise values of the $b_{i}$ at present (see Table 2 in [12]).

In summary, the main conclusion from Fig. 1 is that it is possible to obtain an improved description of $\pi N$ scattering including the $\Delta$ (1232), with the $a_{i}$ values obtained from resonance saturation. Note however, that the $S_{31}$ phase shift does not have any real improvement. For illustration we also give in Fig. 1 the extrapolation of the $O\left(q^{3}\right)$ HBChPT results to high energies (dotted line) as well as the IAM result (dashed line), using the $a_{i}$ and $b_{i}$ values in [9].

(b) Unconstrained IAM fits. Of course, we can get much better fits (all with $\chi^{2} / \mathrm{DOF} \leqslant 1$ ) by leaving all the parameters free. For illustration, see the dashed-dotted line in Fig. 1. There are again strong correlations and the actual value of each one of the $\hat{a}_{i}$ and $\hat{b}_{i}$ could be extremely unnatural. The correlations now are even more complicated due to the quadratic $t_{2}^{2}$ term in the denominator of Eq. (4). By inspection of the analytic formulas, we find that the $\hat{a}_{1}+\hat{a}_{2}, \hat{a}_{5}-4 \hat{a}_{1}$, $2\left(\hat{b}_{1}+\hat{b}_{2}\right)+\left(\hat{b}_{16}-\hat{b}_{15}\right)$ combinations are the most relevant, and remain rather stable for these fits. However it is not possible to obtain a meaningful determination of each individual parameter without any other additional assumption (like resonance saturation). That is again due to the large number of parameters, but also to the slow HBChPT convergence.

(c) The $\Delta(1232)$ resonance. The IAM generates dynamically a pole in the second Riemann sheet at $\sqrt{s}=(1212$ $-i$ 47) $\mathrm{MeV}$, which is rather stable within all the fits and in very good agreement with the data [20].

(d) Threshold parameters. For definitions and notation we refer again to [9]. Our results are shown in Table II, where we have also listed the experimental values, extracted from [10]. As pointed out in [10] and [9], the errors for those values are clearly underestimated. Hence, it should be borne
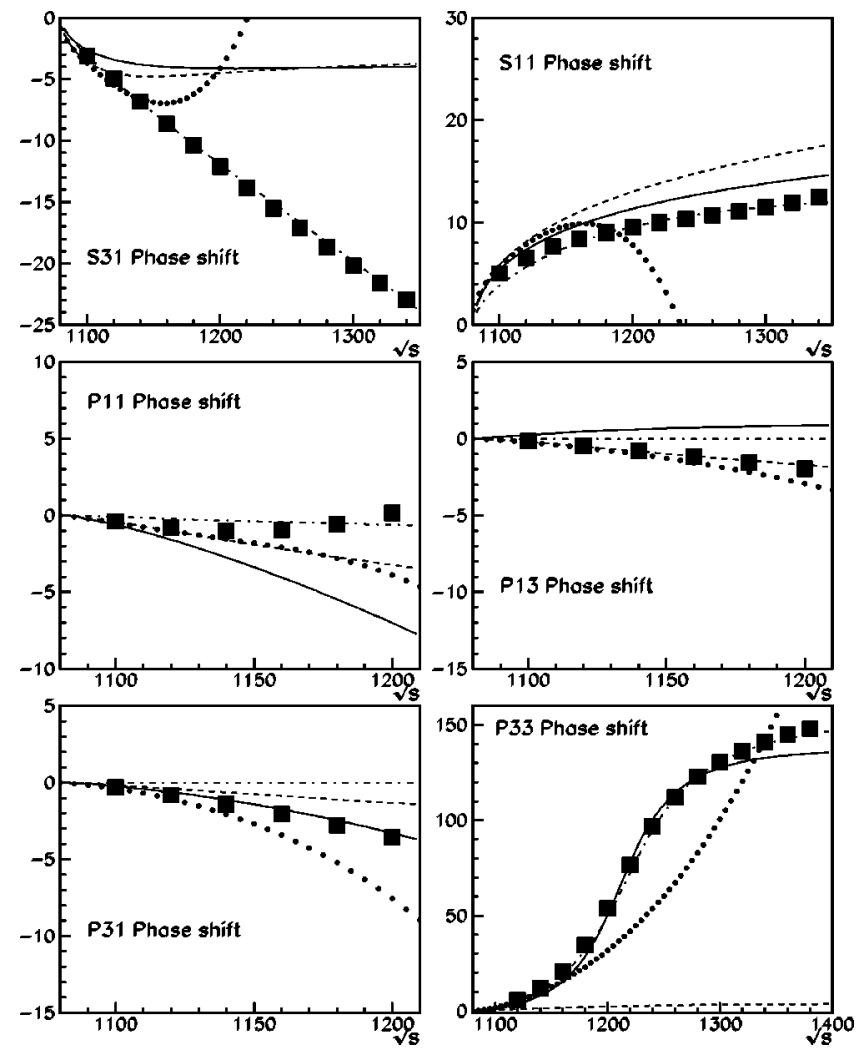

FIG. 1. $\pi-N$ scattering phase shifts. The dotted curve is the extrapolated HBChPT result, with the chiral parameters of [9]. The dashed line is the IAM with the same parameters, and the continuous line is the IAM constrained to resonance saturation (fit 2, see text). The dashed-dotted line is one unconstrained IAM fit. The data come from [11]. 
TABLE II. $\pi N$ threshold values with different IAM fits, and their experimental values (from [10], see text).

\begin{tabular}{lccc}
\hline \hline & $\begin{array}{c}\text { Extrapolated } \\
\text { from } \\
\text { experiment }\end{array}$ & $\begin{array}{c}\text { IAM constrained } \\
\text { to resonance } \\
\text { saturation (fit 2) }\end{array}$ & $\begin{array}{c}\text { IAM } \\
\text { unconstrained }\end{array}$ \\
\hline$a_{0}^{+}\left(\mathrm{GeV}^{-1}\right)$ & $-0.07 \pm 0.01$ & 0.02 & -0.12 \\
$a_{0}^{-}\left(\mathrm{GeV}^{-1}\right)$ & $0.67 \pm 0.1$ & 0.72 & 0.53 \\
$b_{0}^{+}\left(\mathrm{GeV}^{-3}\right)$ & $-16.9 \pm 2.5$ & -2.8 & -16.43 \\
$b_{0}^{-}\left(\mathrm{GeV}^{-3}\right)$ & $5.1 \pm 2.3$ & -9.0 & 13.59 \\
$a_{1+}^{+}\left(\mathrm{GeV}^{-3}\right)$ & $50.5 \pm 0.5$ & 41.04 & 79.08 \\
$a_{1-}^{+}\left(\mathrm{GeV}^{-3}\right)$ & $-21.6 \pm 0.5$ & -18.35 & -2.09 \\
$a_{1+}^{-}\left(\mathrm{GeV}^{-3}\right)$ & $-31.0 \pm 0.6$ & -11.52 & -39.58 \\
$a_{1-}^{-}\left(\mathrm{GeV}^{-3}\right)$ & $-4.4 \pm 0.4$ & -6.75 & -2.00 \\
\hline \hline
\end{tabular}

in mind that the threshold parameters are not so well determined as it may seem from those errors (see also [7]). Our fits give a reasonably good agreement with experiment for the $S$-wave scattering lengths. For most $P$-waves, we agree with the order of magnitude and sign. Our results are also in rough agreement with [7], where they give $-0.07 \mathrm{GeV}^{-1}$ $\leqslant a_{0}^{+} \leqslant 0.04 \mathrm{GeV}^{-1}$ and $0.6 \mathrm{GeV}^{-1} \leqslant a_{0}^{-} \leqslant 0.67 \mathrm{GeV}^{-1}$.

\section{B. Conclusions and discussion}

We have unitarized the HBChPT $\mathcal{O}\left(q^{3}\right) \pi N$ elastic scattering amplitude with the inverse amplitude method. This approach is able to describe the phase shifts up to the inelastic thresholds and, in addition, it gives the correct pole for the $\Delta$ (1232) in the $P_{33}$ channel. Our fits use the extrapolated phase shifts between $\sqrt{s}=1130 \mathrm{MeV}$ and the corresponding inelastic thresholds. Within this approach, we can predict the thresholds values, which for the $S$ waves are in good agreement with experiment and with recent determinations.

Unfortunately, since there are large correlations between some parameters, it is possible to obtain good fits with very different sets of parameters, which can have rather unphysical values. This is due to different reasons: (a) The slow convergence of the series, since contributions from different orders are comparable in almost every partial wave. The effect of higher order terms, which was less relevant at threshold, is absorbed in our case in the values of the chiral coefficients. (b) There are strong correlations between the parameters, and the fits are only sensitive to certain combinations. Hence the values of each individual coefficient are meaningless.

The most relevant conclusion of this study is that we can still reproduce the $\Delta(1232)$ with the $a_{i}$ values expected from the resonance saturation hypothesis, keeping a reasonably good description for the other channels.

Finally, we would like to remark that the method developed here can be easily extended to the case of $S U(3)$ symmetry as well as to the coupled channel formalism. Further work along these lines is in progress.

\section{ACKNOWLEDGMENTS}

This work was partially supported by DGICYT under contract AEN97-1693 and PB98-0782. J.R.P. thanks J.A. O1ler and E. Oset for useful discussions.
[1] E. Jenkins and A. V. Manohar, Phys. Lett. B 255, 558 (1991); V. Bernard, N. Kaiser, J. Kambor, and U. -G. Meissner, Nucl. Phys. B388, 315 (1992); G. Ecker, Czech. J. Phys. 44, 405 (1994); V. Bernard, N. Kaiser, and U. -G. Meissner, Int. J. Mod. Phys. E 4, 193 (1995).

[2] E. Jenkins and A. V. Manohar, Phys. Lett. B 259, 353 (1991).

[3] S. Weinberg, Physica A 96, 327 (1979); J. Gasser and H. Leutwyler, Ann. Phys. (N.Y.) 158, 142 (1984); Nucl. Phys. B250, 463 (1985).

[4] J. Gasser, M. E. Sainio, and A. Svarc, Nucl. Phys. B307, 779 (1988).

[5] T. Mannel, W. Roberts, and Z. Ryzak, Nucl. Phys. B368, 315 (1992).

[6] G. Ecker and M. Mojzis, Phys. Lett. B 365, 312 (1996).

[7] N. Fettes, U.-G. Meissner, and S. Steininger, Nucl. Phys. A640, 199 (1998).

[8] V. Bernard, N. Kaiser, and U.-G. Meissner, Phys. Lett. B 309, 421 (1993); Phys. Rev. C 52, 2185 (1995); Phys. Lett. B 389, 144 (1996); Nucl. Phys. A615, 483 (1997).

[9] M. Mojzis, Eur. Phys. J. C 2, 181 (1998).

[10] R. Koch and E. Pietarinen, Nucl. Phys. A336, 331 (1980).
[11] R. Arndt et al., nucl-th/9807087. SAID online-program (Virginia Tech Partial-Wave Analysis Facility). Latest update, http://said.phys.vt.edu

[12] P. Büttiker and U.-G. Meissner, Nucl. Phys. A668, 97 (2000).

[13] A. Datta and S. Pakvasa, Phys. Rev. D 56, 4322 (1997); P. J. Ellis and H.-B. Tang, Phys. Rev. C 57, 3356 (1998).

[14] U-G. Meissner and J. A. Oller, nucl-th/9912026.

[15] T. N. Truong, Phys. Rev. Lett. 61, 2526 (1988); 67, 2260 (1991); A. Dobado, M. J. Herrero, and T. N. Truong, Phys. Lett. B 235, 134 (1990); A. Dobado and J. R. Peláez, Phys. Rev. D 47, 4883 (1993); 56, 3057 (1997).

[16] J. A. Oller, E. Oset, and J. R. Peláez, Phys. Rev. Lett. 80, 3452 (1998); Phys. Rev. D 59, 074001 (1999); hep-ph/9909556; F. Guerrero and J. A. Oller, Nucl. Phys. B537, 459 (1999).

[17] T. Ericson and W. Weise, Pions and Nuclei (Oxford, New York, 1988) and references therein.

[18] J. L. Basdevant, Fortschr. Phys. 20, 283 (1972).

[19] E. Oset and A. Ramos, Nucl. Phys. A635, 99 (1998).

[20] Review of Particle Physics, Particle Data Group, C. Case, et al., Eur. Phys. J. C 3, 1 (1998). 